\title{
Alteraciones Morfológicas en Dientes Sometidos a Altas Temperaturas con Interés Forense
}

\author{
Morphological Changes in Teeth Exposed to High Temperatures with Forensic Purposes
}

Leticia Rubio; José Manuel Sioli*; Ignacio Santos*; Gabriel M. Fonseca** \& Stella Martin-de-las-Heras ${ }^{* * *}$

RUBIO, L.; SIOLI, J. M.; SANTOS, I.; FONSECA, G. M. \& MARTIN-DE-LAS-HERAS, S. Alteraciones morfológicas en dientes sometidos a altas temperaturas con interés forense. Int. J. Morphol., 34(2):719-728, 2016.

RESUMEN: Los dientes son los elementos más resistentes del esqueleto humano y son utilizados en muchas ocasiones en la rutina de los laboratorios forenses. El objetivo de este estudio fue describir los cambios dentarios estructurales, morfológicos y del color que se producen tras la incineración, para ayudar en el manejo adecuado de las muestras en un escenario forense. Setenta dientes fueron expuestos a intervalos de temperatura de $100^{\circ} \mathrm{C} \mathrm{a} 1200^{\circ} \mathrm{C}$, durante $60 \mathrm{~min}$. Un grupo control de 10 dientes no fue expuesto al choque térmico. Después de la incineración, los dientes fueron fotografiados y radiografiados para evaluar visualmente los cambios morfológicos. La medida del cambio de color fue realizada mediante un espectrofotómetro. Los resultados mostraron cambios estructurales y del color, así como alteraciones radiológicas, que se relacionaron con el intervalo de temperatura a la que fueron incinerados los dientes. En conclusión, es posible informar sobre la temperatura aproximada de calcinación de los dientes basándose en los cambios fotográficos, radiográficos y del color.

PALABRAS CLAVE: Diente calcinado; Cambios color; Rayos-X; Morfología; Espectrofotometría.

\section{INTRODUCCIÓN}

Los dientes son considerados las estructuras más resistentes del cuerpo humano, pudiendo soportar temperaturas de hasta $1600{ }^{\circ} \mathrm{C}$ sin una pérdida importante de su microestructura (Moreno et al., 2009), permaneciendo casi intactos tiempo después de que los tejidos blandos y esqueléticos han sido destruidos por la incineración. Otra característica a tener en cuenta es que los dientes se encuentran articulados en el hueso alveolar del maxilar y de la mandíbula, tejidos óseos que, unidos a los tejidos blandos, mucosos, epiteliales y musculares que los rodean, les proporcionan una mayor protección en caso de exposición a altas temperaturas (Myers et al., 1999; Fereira et al., 2008).

El análisis de los dientes sometidos a altas temperaturas constituye un aspecto fundamental en el campo de las ciencias forenses. Una gran variedad de acontecimientos se producen en los que el fuego actúa como protagonista: accidentes aéreos, de tráfico, terremotos, atentados con bombas o cremaciones ilícitas. Además, el fuego puede ser empleado para destruir pruebas forenses en casos criminales, a menudo tratando de evitar la identificación y recuperación de la víctima
(Savio et al., 2006). En estos casos, los dientes de los cadáveres carbonizados pueden aportar valiosa información sobre la identificación del individuo, pero también de las circunstancias que rodean al fuego, la temperatura alcanzada en el lugar de los hechos, la fragilidad para la recuperación de la muestra, la degradación del ADN, etc. (Pol et al., 2015).

La exposición a altas temperaturas produce una evaporación del agua y del material orgánico, que conlleva a un proceso de contracción de los tejidos dentales. Este fenómeno va a ocasionar la aparición de una serie de cambios morfológicos en el diente, tanto a nivel estructural, como en su coloración. En los últimos años, se han descrito algunas técnicas para analizar estas alteraciones como la radiografía (Savio et al.), histología (Myers et al.; Fereira et al.), microscopía electrónica de barrido (Muller et al., 1998; Merlati et al., 2002; Karkhanis et al., 2009; Sandholzer et al., 2013) y estéreo-microscopía (Merlati et al., 2004; Moreno et al.). No obstante, serían necesarias nuevas investigaciones que abordaran situaciones prácticas forenses usando una tecnología accesible en dichos casos.

\footnotetext{
* Departamento de Medicina Legal y Forense, Universidad de Málaga, Málaga, España.

** Centro de Investigación en Morfología Aplicada (CIMA), Facultad de Odontología, Universidad de La Frontera, Temuco, Chile.

**** Departamento de Medicina y Odontología Legal y Forense, Universidad de Granada, Granada, España.
} 
La mayoría de las investigaciones sobre las alteraciones en el color de dientes sometidos a altas temperaturas han sido realizadas de forma visual (Muller et al.; Moreno et al.; Karkhanis et al.; Sandholzer et al., 2013) o, en algunos casos, mediante la comparación con guías de color (Muller et al.; Karkhanis et al.). Sin embargo, para una mayor objetividad en la valoración del color, varios autores recomiendan la utilización de técnicas colorimétricas que proporcionan una evaluación de la coloración precisa y reproducible (American Society for Testing and Materials, 1993; Paul et al., 2002; Martín de las Heras et al., 2003).

El objetivo principal de este trabajo fue estudiar los cambios estructurales macroscópicos y de la coloración de dientes sometidos a altas temperaturas con interés forense, mediante el empleo de lupas, fotografías digitales, radiografías digitales y espectrofotometría.

\section{MATERIAL Y MÉTODO}

Muestra y preparación. La muestra consistió en 80 dientes permanentes humanos, premolares y molares, extraídos por motivos clínicos válidos (enfermedad periodontal o tratamiento de ortodoncia), los cuales fueron obtenidos en el Servicio Público de Salud Oral de Cádiz (España). La investigación fue aprobada por el Comité de Ética para la Investigación en Seres Humanos de la Universidad de Málaga (España). Los pacientes fueron 47 mujeres y 33 hombres, con edades que oscilaron entre los 19 y 87 años, siendo la edad media de 47,9 años. Todos los dientes seleccionados cumplieron el criterio de estar sanos (libres de caries, sin tratamientos odontológico previos, sin tinciones exógenas o endógenas). Los dientes fueron extraídos de manera mínimamente invasiva preservando la total integridad de sus estructuras tisulares, luego fueron lavados con agua estéril, preservados y enviados al laboratorio para su análisis. Finalmente, las muestras fueron aleatorizadas en 8 grupos de 10 dientes cada uno.

Incineración de las muestras. Los dientes fueron sometidos al calor en un horno tipo mufla (Nabertherm LT 40/ 12, Nabertherm GmbH, Alemania) a temperaturas de 100 , $200,400,600,800,1000$ y $1200^{\circ} \mathrm{C}$, respectivamente. Un grupo control de 10 dientes no fue expuesto al choque térmico. Los dientes fueron colocados individualmente en crisoles de alúmina al 99,9\% y, partiendo de una temperatura ambiente, se empleó una tasa de ascenso de 10 ${ }^{\circ} \mathrm{C}$ por minuto. Una vez alcanzado el intervalo de temperatura deseado, se incineraron durante $60 \mathrm{~min} y$, a continuación, se dejaron enfriar hasta llegar a una temperatura ambiente.
Análisis morfológico visual de la estructura dental y del color. El examen visual de las alteraciones estructurales y del color fue realizado por un observador experto, con luz natural, utilizando lupas de aumento de 2,5X (Orascoptic ${ }^{\circledR}$, Middleton, Winsconsin, USA). La inspección visual se complementó con fotografías captadas con una cámara réflex digital (Canon $500 \mathrm{D}^{\circledR}$, Canon Inc. Tokyo, Japón), equipada con un objetivo macro de 100 $\mathrm{mm}$ y un flash anular (Canon MR-14 Ex ${ }^{\circledR}$, Canon Inc, Tokyo, Japón). Las imágenes se visualizaron con el programa Adobe Camara Raw 7.3 ${ }^{\circledR}$ (Adobe Systems incorporated, San Jose, California, USA), con el modo zoom hasta un $100 \%$.

Análisis Radiográfico. Las alteraciones estructurales radiográficas fueron analizadas mediante radiografías periapicales, usando la técnica de cono largo. Se empleó un equipo de radiografía digital intraoral (Carestream $2200^{\circledR}$ ) y un sistema de radiografía digital RVG 6100 (Carestream ${ }^{\circledR}$, Nueva York, USA). Para facilitar el manejo y posicionar correctamente los dientes se diseñaron unos moldes de silicona de condensación (Coltoflax ${ }^{\circledR}$, Coltene Whaledent, Alstätten, Suiza). El equipo fue calibrado previamente para que todas las radiografías se realizaran a igual dosis de $0,31 \mathrm{Kv}$.

Análisis del color mediante espectrofotometría. El color dental fue medido en la cara bucal de los dientes con un espectrofotómetro portátil (Spectrocolor ${ }^{\circledR}$, Dr Lange, Keison Products Co, Essex, Inglaterra). Los parámetros utilizados fueron D65 para la iluminación y $8^{\circ}$ para el observador estándar. El espectrofotómetro era calibrado antes de cada sesión y se realizaban tres registros de cada muestra, utilizando el valor promedio de las mismas.

Los valores colorimétricos $\left(\mathrm{L}^{*} \mathrm{a}^{*}\right.$ y b*), así como la luminancia (Y; unidades: $\mathrm{cd} / \mathrm{m} 2$; candela/metro cuadrado) fueron obtenidos conforme al sistema CIELAB. El valor $\mathrm{L}^{*}$ es una medida de la luminosidad de un objeto en una escala que va del 0 (negro) al 100 (blanco). El valor $\mathrm{a}^{*}$ es la medida que va del rojo (valores de $\mathrm{a}^{*}$ positivos) al verde (valores negativos de $\mathrm{a}^{*}$ ). El valor $\mathrm{b}^{*}$ es la medida que va del amarillo (valores positivos de $\mathrm{b}^{*}$ ) al azul (valores negativos de $b^{*}$ ). Los valores $a^{*} y^{*}$ se aproximan al valor 0 para colores neutrales, aumentando su magnitud para colores más saturados. Para evaluar el amarilleamiento y blancura de los dientes, los índices de blancura (WI) y amarilleamiento (YI) fueron calculados con las fórmulas propuestas por la Sociedad Americana para Pruebas y Materiales, ASTM (American Society for Testing and Materials).

$$
\mathrm{WI}=4 \mathrm{Z} \%-\text { Yrel YI= } 100(1-0,847 \mathrm{Z} / \mathrm{Yrel})
$$


Donde Z\% fue calculada como: Z\%= (Z/Zn) 100; Z es el tri-estímulo $Z$ de la muestra; y $Z n$ el tri-estímulo del blanco estándar; y Yrel es el factor de luminancia, calculado como: Yrel $=100(\mathrm{Y} / \mathrm{Yn})$, donde $\mathrm{Y}$ es la luminancia de la muestra y Yn la luminancia del blanco estándar.

\section{RESULTADOS}

Análisis visual de la estructura dental. Los cambios estructurales producidos en la corona y en la raíz de los dientes sometidos a altas temperaturas están representados en las Figuras 1 y 2, respectivamente. Las fisuras longitudinales y transversales comenzaron a $\operatorname{los} 100^{\circ} \mathrm{C}$, tanto a nivel coronal como radicular. A este intervalo de temperatura, todas las muestras presentaron cambios a nivel coronal, sin embargo, un $30 \%$ de las mismas se mantuvieron intactas a nivel radicular. Las grietas aparecieron a los $100^{\circ} \mathrm{C}$ en el cemento y a los $400{ }^{\circ} \mathrm{C}$ en el esmalte. El esmalte adquirió un aspecto cuarteado a los $600{ }^{\circ} \mathrm{C}$, mientras el cemento lo hizo a los $400{ }^{\circ} \mathrm{C}$ de temperatura. La fractura o "estallido" del esmalte y la separación del esmalte y la dentina en la zona cervical, se produjo entre $\operatorname{los} 200^{\circ} \mathrm{C}$ y $400{ }^{\circ} \mathrm{C}$. No obstante, la separación del esmalte en forma de "casquete" comenzó a $\operatorname{los} 400{ }^{\circ} \mathrm{C}$, y las primeras fracturas y separaciones de la corona y la raíz a los $600^{\circ} \mathrm{C}$. A esta misma temperatura se inició la fragmentación del esmalte, mientras que en el cemento aconteció mucho antes, a los $100^{\circ} \mathrm{C}$ de temperatura.

Análisis visual de la coloración dental. La evolución del color del diente sometido a altas temperaturas puede ser observado en la Figura 3. En la Figura 4 se muestran los cambios del color de la corona de los dientes para cada intervalo de temperatura. A los $100{ }^{\circ} \mathrm{C}$, el color de la corona evolucionó a un marrón pálido, que se tornó algo más marcado a los $200^{\circ} \mathrm{C}$, posteriormente, se transformó en un marrón grisáceo $\left(400^{\circ} \mathrm{C}\right)$, gris oscuro con pequeñas manchas azuladas $\left(600^{\circ} \mathrm{C}\right)$, gris más oscuro con manchas blancas $\left(800{ }^{\circ} \mathrm{C}\right)$, blanco con manchas grises $\left(1000{ }^{\circ} \mathrm{C}\right)$ y blanco "tiza" con o sin manchas azuladas $\left(1200^{\circ} \mathrm{C}\right)$. Los cambios de coloración en la raíz dental fueron más homogéneos que a nivel coronal en cada uno de sus grupos (Fig. 5). El color radicular adquirió un tono blanco-amarillento opaco (100 $\left.{ }^{\circ} \mathrm{C}\right)$, marrón amarillento $\left(200{ }^{\circ} \mathrm{C}\right)$, marrón oscuro con zonas negras $\left(400{ }^{\circ} \mathrm{C}\right)$, blanco con manchas grises $\left(600^{\circ} \mathrm{C}\right) \mathrm{y}$, finalmente, blanco "tiza" (800-1200 $\left.{ }^{\circ} \mathrm{C}\right)$.

Análisis radiográfico dental. El análisis morfológico radiográfico a nivel coronal reveló la aparición de fisuras entre el esmalte y la dentina a temperaturas entre $200^{\circ} \mathrm{C}$ y $400{ }^{\circ} \mathrm{C}$, añadiendo fisuras en el interior de la dentina desde los $200{ }^{\circ} \mathrm{C}$ a los $1200{ }^{\circ} \mathrm{C}$. La reducción a fragmentos de la corona dental ocurrió entre los $600{ }^{\circ} \mathrm{C}$ y $1200{ }^{\circ} \mathrm{C}$ (Fig. 6). Las alteraciones en las raíces dentales aparecieron después que las de nivel coronal, no evidenciándose cambio alguno sino hasta $\operatorname{los} 200{ }^{\circ} \mathrm{C}$ en casi la totalidad de las muestras. Las fisuras, a excepción de un caso, aparecieron a los 400 ${ }^{\circ} \mathrm{C}$, mientras que las fracturas no se observaron sino hasta los $1000-1200{ }^{\circ} \mathrm{C}$ de temperatura (Fig. 7). La evolución de las alteraciones radiográficas de los dientes sometidos a altas temperaturas pueden observarse en la Figura 8.

Análisis del color dental con espectrofotometría. La Figura 9 muestra los curvas colorimétricas de la corona de los dientes sometidos a altas temperaturas. Los valores de $\mathrm{L}^{*}$ disminuyeron desde el grupo de $100{ }^{\circ} \mathrm{C}$ hasta el grupo de $400{ }^{\circ} \mathrm{C}$ de temperatura, sin embargo, a $600{ }^{\circ} \mathrm{C}$ los valores aumentaron alcanzando un nivel similar al grupo control (sin incinerar). A $800{ }^{\circ} \mathrm{C}$ los valores volvieron a reducirse, pero

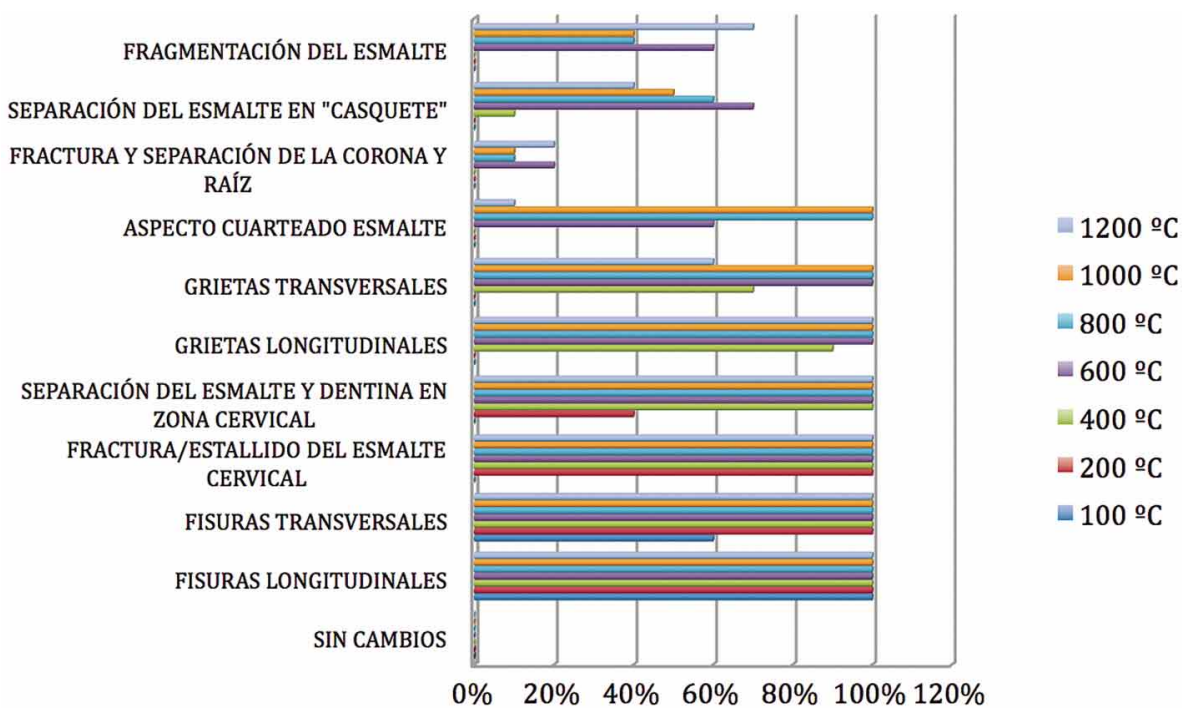

Fig. 1. Cambios estructurales acontecidos en la corona de dientes sometidos a altas temperaturas. 


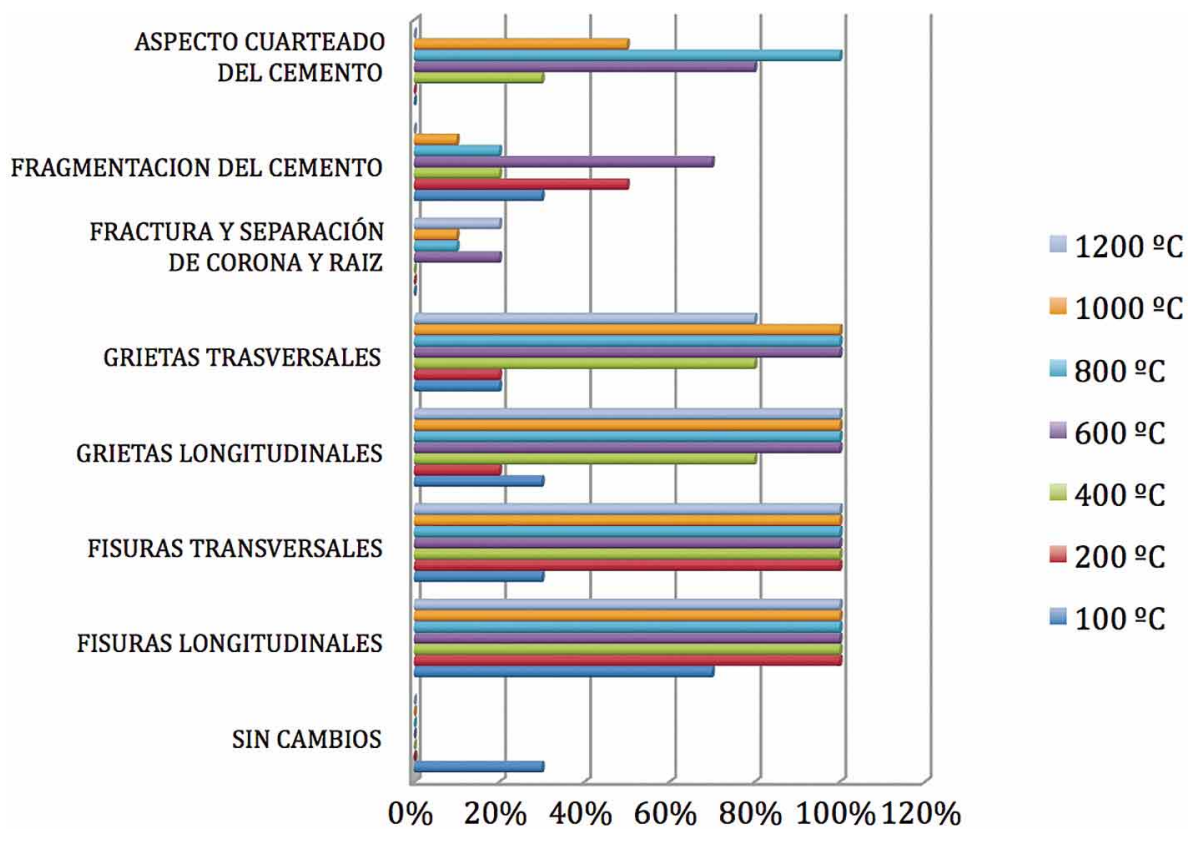

Fig. 2. Cambios estructurales acontecidos en la raíz de dientes sometidos a altas temperaturas.

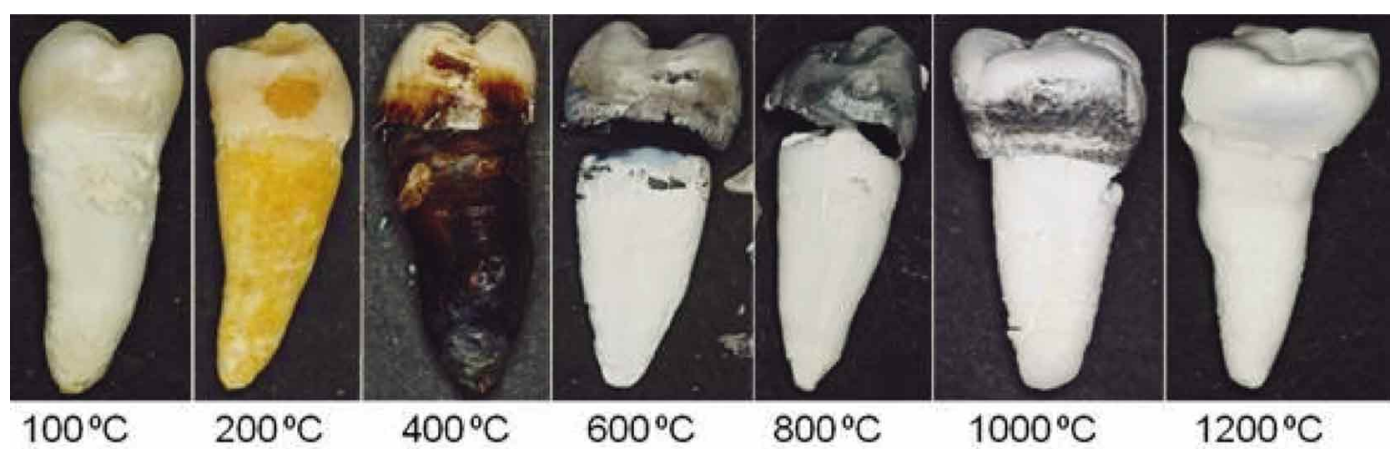

Fig. 3. Evolución del color del diente sometido a altas temperaturas.

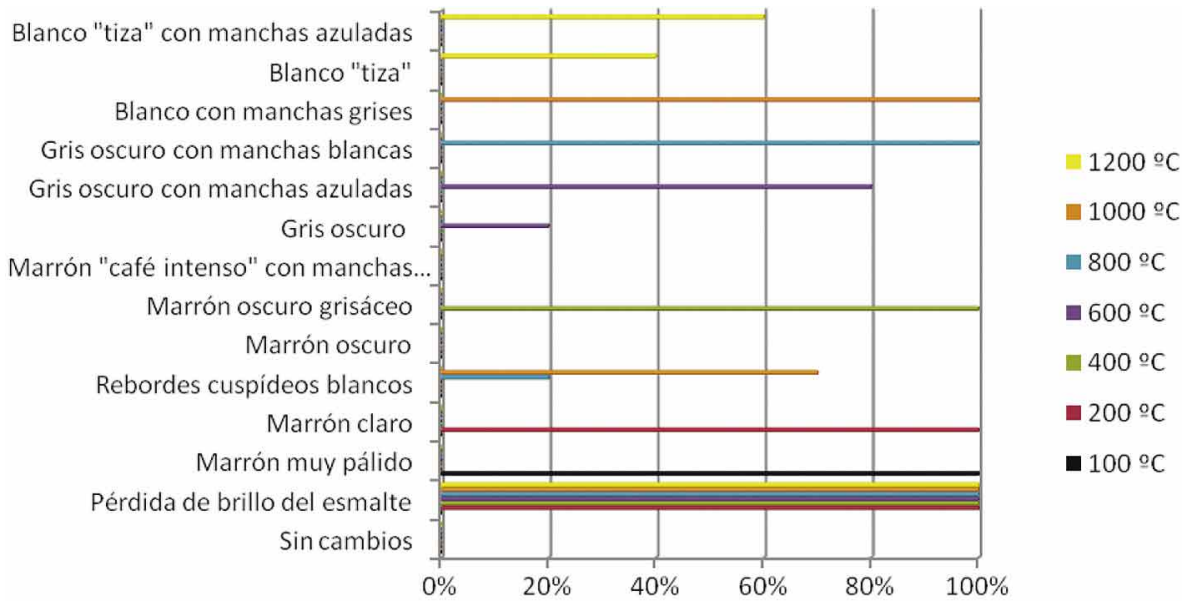

Fig. 4. Cambios de coloración en la corona de dientes sometidos a altas temperaturas detectados por inspección visual. 
esta vez de forma leve. Los valores más altos de luminosidad fueron obtenidos a $1000^{\circ} \mathrm{C}$ y $1200^{\circ} \mathrm{C}$. La cromaticidad $\mathrm{a}^{*}$ evolucionó de la siguiente forma: de $0{ }^{\circ} \mathrm{C}$ a $100{ }^{\circ} \mathrm{C}$ sufrió un descenso muy marcado de sus valores, moviéndose desde el color rojo a un verde más saturado. A $200{ }^{\circ} \mathrm{C}, 400^{\circ} \mathrm{C}$ y $1200^{\circ} \mathrm{C}$ las muestras adquirieron un color rojo neutro, mientras que se alcanzó un color verde a $100{ }^{\circ} \mathrm{C}, 600{ }^{\circ} \mathrm{C}, 800{ }^{\circ} \mathrm{C}$ y $1000^{\circ} \mathrm{C}$. El color verde más saturado se obtuvo en el grupo de $100^{\circ} \mathrm{C}$ de temperatura. La cromaticidad $b^{*}$ disminuyó desde los $100{ }^{\circ} \mathrm{C}$ hasta los $600{ }^{\circ} \mathrm{C}$, presentando un valor positivo que se movió de un color amarillo más saturado a un amarillo neutro. A los $800{ }^{\circ} \mathrm{C}, 1000{ }^{\circ} \mathrm{C}$ y $1200{ }^{\circ} \mathrm{C}$ los valores siguieron descendiendo, pasando a ser negativos y, por tanto, hacia un color más azulado. Con respecto a la blancura (WI), se registraron valores similares hasta los 400 ${ }^{\circ} \mathrm{C}$, sin embargo, a partir de los $600{ }^{\circ} \mathrm{C}$ los valores fueron incrementándose hasta $\operatorname{los} 1200^{\circ} \mathrm{C}$. Los valores más alto de WI fueron encontrados a $1000^{\circ} \mathrm{C}$ y $1200^{\circ} \mathrm{C}$. Los valores de amarilleamiento (YI) disminuyeron desde los $100{ }^{\circ} \mathrm{C}$ hasta los $800{ }^{\circ} \mathrm{C}$, siendo en esta última temperatura a la que se obtiene el valor más bajo. A $1000^{\circ}$ y $1200^{\circ}$ el amarilleamiento aumentó con respecto a los $800^{\circ} \mathrm{C}$, aunque continuó en valores negativos.

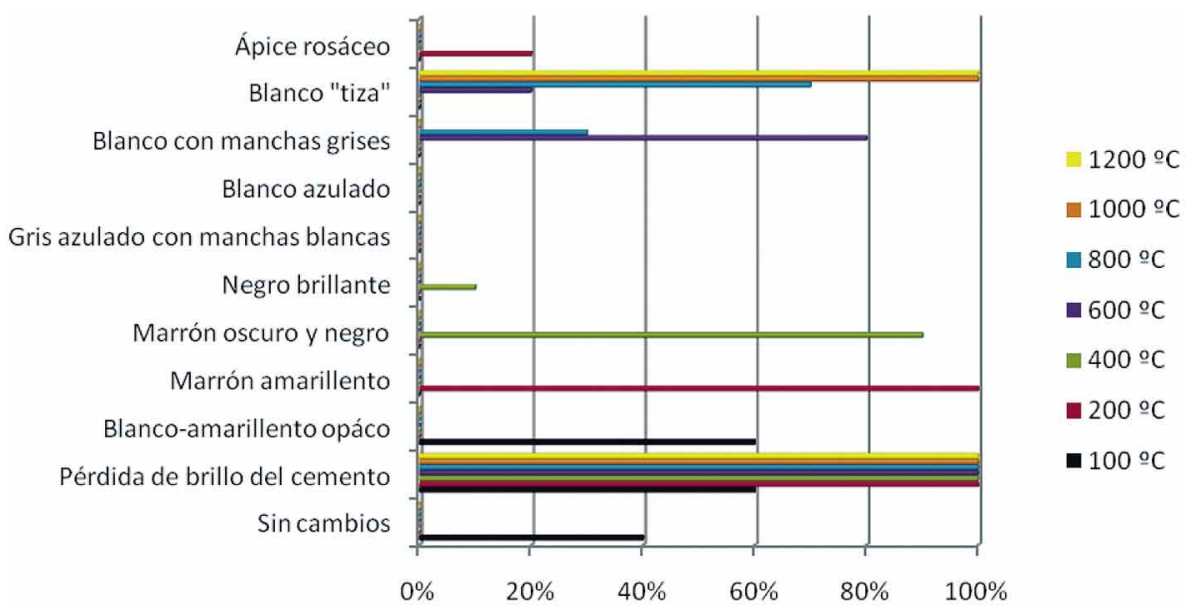

Fig. 5. Cambios de coloración en la raíz de dientes sometidos a altas temperaturas detectados por inspección visual.

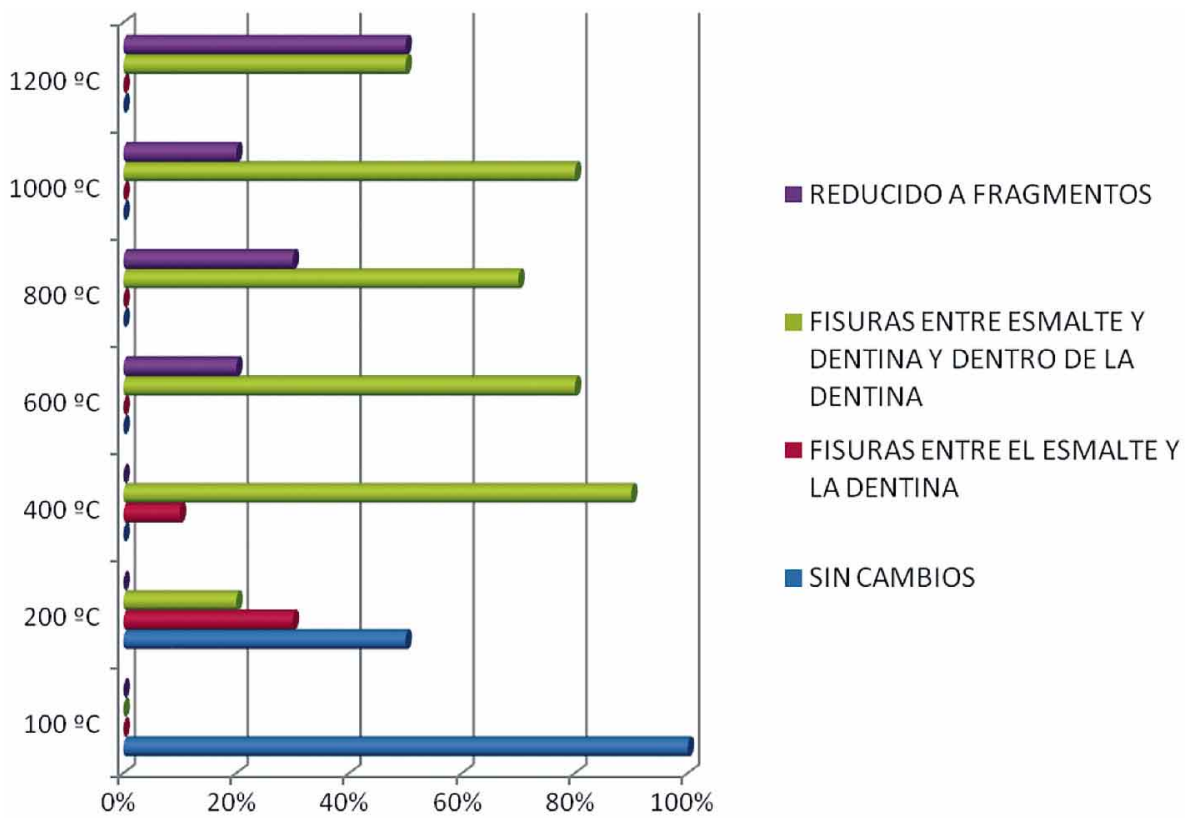

Fig. 6. Cambios estructurales radiográficos en la corona de dientes sometidos a altas temperaturas. 


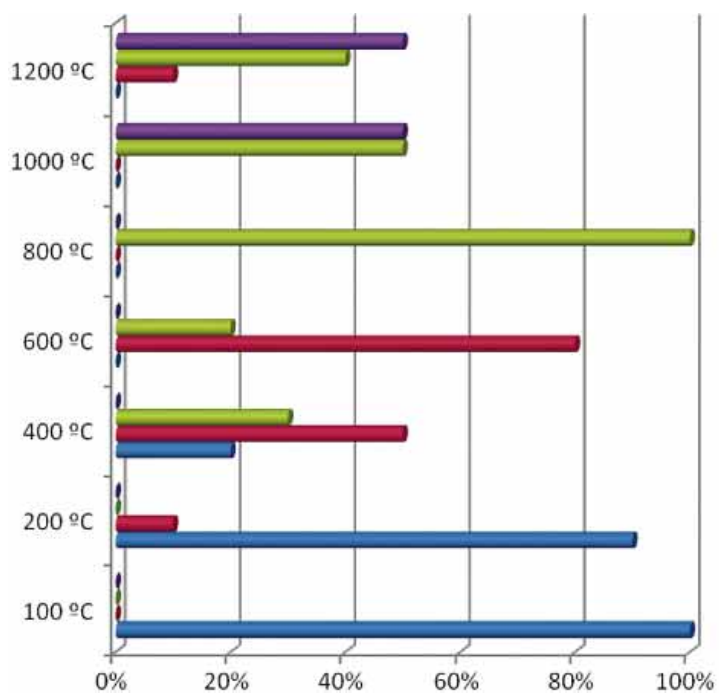

- FRACTURASLARGASA TRAVES DE LA DENTINA

IISURASA TRAVES DE LA DENTINA

- FISURAS DENTRO DE LA DENTINA

- SIN CAMBIOS
Fig. 7. Cambios estructurales radiográficos en la raíz de dientes sometidos a altas temperaturas.

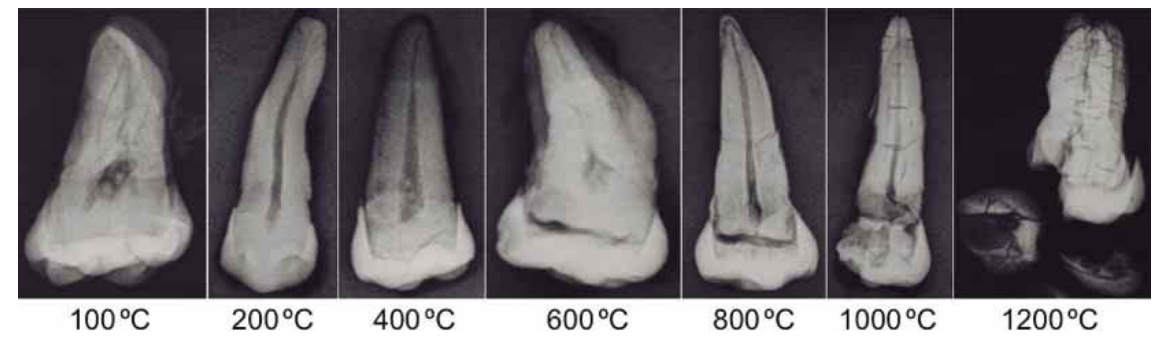

Fig. 8. Evolución de las alteraciones estructurales radiográficas de dientes sometidos a altas temperaturas.

A

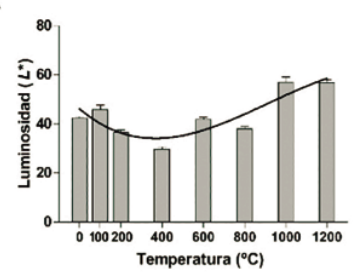

C

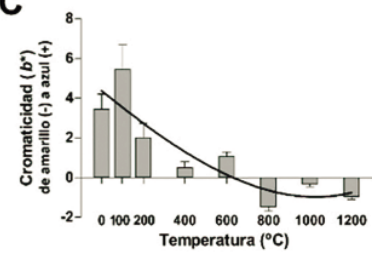

B

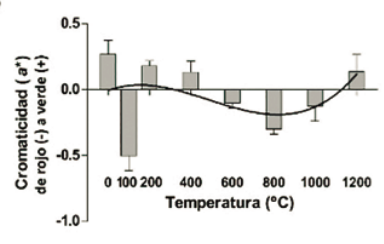

D

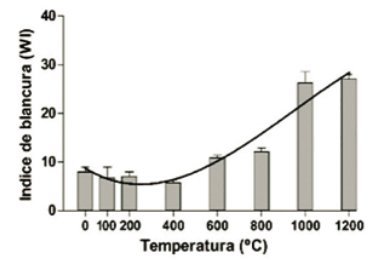

E

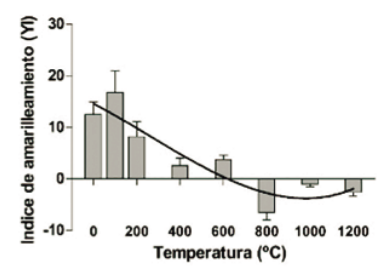




\section{DISCUSIÓN}

El diente es una muestra clave y, a menudo la única disponible, en la resolución de casos forenses, siendo de especial relevancia en la identificación de víctimas carbonizadas y en las circunstancias que rodean el fuego. En este trabajo de investigación fueron analizados dientes posteriores, a pesar de que los anteriores han sido utilizados en muchos estudios (Myers et al.; Merlati et al., 2002, 2004; Savio et al.; Beach et al., 2008; Moreno et al.; Karkhanis et al.; Patidar et al., 2010). Esta preferencia es compartida por otros autores (Merlati et al., 2004; Karkhanis et al.; Rubio et al., 2009; Sandholzer et al., 2013) ya que los dientes posteriores suelen resistir más el choque térmico gracias a la protección que le brindan la piel, la mucosa, los músculos y los tejidos adiposo y óseo (Savio et al.), mientras que los dientes anteriores suelen presentar un mayor daño térmico debido a su exposición al fuego cuando los labios se retraen, (Karkhanis et al.). Por otra parte, el mayor volumen de pulpa dental de los dientes posteriores los hace beneficiosos como muestra, ya que proporcionan una mejor fuente de ADN (Rubio et al., 2009).

La ausencia de protocolos sobre incineración de las muestras, provoca una gran heterogeneidad de los estudios reportados a la fecha. Las temperaturas empleadas por otros investigadores fueron muy similaresa las utilizadas en este estudio, aunque no idénticas (Muller et al.; Merlati et al., 2002, 2004; Savio et al.; Karkhanis et al.; Moreno et al.; Sandholzer et al., 2013). También se han aplicado temperaturas únicas, como son $400{ }^{\circ} \mathrm{C}$ y $1100{ }^{\circ} \mathrm{C}$ (Patidar et al.), $900{ }^{\circ} \mathrm{C}$ (Bonavilla et al., 2008) y $1150{ }^{\circ} \mathrm{C}$ (Pol et al.). Otra característica en la que varían mucho los trabajos de investigación analizados, es el tiempo de duración de la incineración. En nuestro caso, una vez alcanzado la temperatura prevista, se mantuvo la incineración durante $60 \mathrm{~min}$, coincidiendo con Muller et al. Otros estudios incineraron durante 30 min (Bonavilla et al.; Sandholzer et al., 2013) e incluso han utilizado diferentes intervalos de tiempo que oscilaron desde los 5 min hasta los 90 min (Muller et al.; Savio et al.; Patidar et al.). Al igual que en este trabajo, la mayoría de los estudios emplearon hornos tipo mufla y no fuego directo para la aplicación de las altas temperaturas (Muller et al.; Merlati et al., 2004; Savio et al.; Fereira et al.; Karkhanis et al.; Moreno et al.; Rubio et al., 2009; Sandholzer et al., 2013). A pesar de que en algunos estudios realizan un precalentamiento del horno hasta alcanzar la temperatura deseada para, posteriormente, introducir las muestras (Muller et al.; Myers et al.; Bonavilla et al.; Karkhanis et al.; Rubio et al., 2009; Patidar et al.; Sandholzer et al., 2013); desde nuestro punto de vista y, coincidiendo con otros autores (Merlati et al., 2002, 2004; Savio et al.; Fereira et al.; Mo- reno et al.), es preferible colocar los dientes en el horno desde temperatura ambiente, para que el incremento de la temperatura se produzca gradualmente, consiguiendo así simular mejor una situación forense real. En nuestro trabajo se empleó una tasa de ascenso de $10^{\circ} \mathrm{C}$ por minuto, como la empleada por Moreno et al. Sin embargo, otras tasas de ascenso utilizadas fueron: $18,8^{\circ} \mathrm{C}$ (Fereira et al.) y $30^{\circ} \mathrm{C}$ por minuto (Merlati et al., 2002, 2004; Savio et al.). Fereira et $a l$. ponen de manifiesto que tasas de ascenso más bajas podrían evitar la explosión de las coronas dentales, coincidiendo con la idea de que a incrementos más graduales el daño térmico será menor.

Los cambios estructurales que sufre el diente sometido al calor, van a depender de la temperatura, del tiempo de exposición pero también de la forma en la que se producen o aplican esas altas temperaturas. Si un diente es quemado gradualmente, el contenido de humedad se evapora poco a poco y el daño de la estructura dental es mínimo; sin embargo, cuando el calor es rápido e intenso, esto va a inducir a una rápida evaporación del contenido orgánico del diente, facilitando la explosión de la corona y la separación de la capa de esmalte, permitiendo así que arda la dentina que está menos mineralizada (Merlati et al., 2002). En nuestra investigación, las fisuras longitudinales y transversales comenzaron a producirse rápido, concretamente a $\operatorname{los} 100^{\circ} \mathrm{C}$, tanto a nivel coronal como radicular (Figs. 1 y 2). Del mismo modo, Karkhanis et al. las observaron a la misma temperatura en un grupo de dientes temporales. Sin embargo, en estudios similares de dientes permanentes, las fisuras están descritas a los $150^{\circ} \mathrm{C}$ (Muller et al.), $200^{\circ} \mathrm{C}$ (Beach et al.) y $400^{\circ} \mathrm{C}$ (Moreno et al.). Las grietas longitudinales y transversales aparecieron muy pronto a nivel radicular y algo más tarde en la corona dental, manifestándose a los $100^{\circ} \mathrm{C}$ y 400 ${ }^{\circ} \mathrm{C}$, respectivamente (Figs. 1 y 2). Otros trabajos de investigación al respecto, las describen a los $300-400{ }^{\circ} \mathrm{C}$ (Muller et al.), entre $371^{\circ} \mathrm{C} \mathrm{y} 427^{\circ} \mathrm{C}$ (Beach et al.) y a los $600{ }^{\circ} \mathrm{C}$ de temperatura (Moreno et al.). El aspecto cuarteado del cemento también se manifestó antes que en el esmalte, a los $400{ }^{\circ} \mathrm{C}$ y $600{ }^{\circ} \mathrm{C}$, respectivamente (Figs. 1 y 2). Este hecho es descrito por Moreno et al. a partir de $l o s 600^{\circ} \mathrm{C}$ en el esmalte y el cemento. La fragmentación del cemento se produjo antes que en el resto de los estudios pudiendo observarla a los $100^{\circ} \mathrm{C}$, sin embargo, la fragmentación del esmalte ocurrió a partir de los $600^{\circ} \mathrm{C}$, coincidiendo con la mayoría de los autores (Muller et al.; Merlati et al., 2004; Beach et al.; Moreno et al.) (Figs. 1 y 2). En nuestro trabajo, muchas de las alteraciones estructurales sucedieron el cemento antes que en el esmalte como consecuencia del mayor contenido inorgánico que éste último posee. Sin embargo, en situaciones reales cabe esperar que las alteraciones radiculares acontezcan algo más tarde puesto que la protección de los tejidos de alrededor no ha sido considerada en 
este trabajo de investigación. Cabe destacar que es la corona dental la que más a menudo proporciona información con interés forense (Terada et al., 2014).

La diferencia en la composición entre el cemento y la dentina no es tan marcada como ocurre a nivel coronal entre el esmalte y la dentina. Esto provoca que el esmalte pierda su escaso contenido en agua y se contraiga rápidamente, produciéndose una separación progresiva entre el esmalte y la dentina que puede llevar hasta la separación completa o fractura del mismo (Muller et al.; Beach et al.). Por tanto, la diferencia existente en el margen de contracción térmica entre el esmalte y la dentina, provoca la separación y/o estallido del esmalte a nivel cervical. En este estudio, al igual que lo reportado por la mayoría de los autores (Muller et al.; Beach et al.; Karkhanis et al.; Moreno et al.), esta alteración comenzó a los $200^{\circ} \mathrm{C}$ (Figs. 1 y 2). A medida que fue progresando esta ruptura, se produjo una separación más evidente del esmalte y de la dentina, algo que ocurre normalmente a $\operatorname{los} 400^{\circ} \mathrm{C}$. Es interesante mencionar que al aumentar la temperatura puede ocurrir un fenómeno en el que el esmalte se desprende completamente de la corona, en forma de "casquete". En nuestro estudio, el primer caso apareció a los $400{ }^{\circ} \mathrm{C}$, aunque no fue sino hasta $\operatorname{los} 600^{\circ} \mathrm{C}$ donde se produjo en la mayoría de los casos (Figs. 1 y 2). Karkhanis et al. informaron este mismo fenómeno a los 400 ${ }^{\circ} \mathrm{C}$ pero en dientes deciduos. Otros reportes lo han descrito a los $427^{\circ} \mathrm{C}$ (Beach et al.), a los $450^{\circ} \mathrm{C}$ (Muller et al.) y a los $800{ }^{\circ} \mathrm{C}$ (Merlati et al., 2004; Moreno et al.). Por último, la fractura y separación de la corona y raíz ocurrió sólo en seis casos, aconteciendo por primera vez en el grupo sometido a $600{ }^{\circ} \mathrm{C}$ de temperatura (Figs. 1 y 2). La escasa presencia de fracturas ante temperaturas tan elevadas puede deberse a la reorganización y fusión de los cristales de hidroxiapatita al rellenar los espacios vacíos dejados por la evaporación del material orgánico y agua, fenómeno conocido como "sinterización" (Shipman et al., 1984).

La microestructura de los huesos y dientes humanos sometidos al efecto del calor ha sido estudiada mediante diferentes técnicas imagenológicas como la dispersión de rayos X de ángulo pequeño (Sandholzer et al., 2014), la difracción de rayos X (Piga et al., 2008), y más recientemente, la micro-tomografía computerizada 3D (Sandholzer et al., 2013). Sin embargo, el alto coste de estas tecnologías las hace inaccesibles para su aplicación en casos prácticos de identificación forense. La escasez de trabajos que utilicen la radiografía convencional en dientes incinerados impide la comparación de nuestros resultados (Figs. 6, 7 \& 8), encontrando tan sólo que la aparición de fisuras y fracturas en la corona y en la raíz se produjeron a temperaturas inferiores a las reportadas por Savio et al. Los dientes soportaron temperaturas de hasta $200^{\circ} \mathrm{C}$ sin prácticamente ninguna alteración estructural visible en la radiografía convencional $\mathrm{y}$, paulatinamente, los cambios radiográficos se fueron haciendo más intensos con el aumento de las temperaturas.

Habitualmente, la determinación del color dental es realizada mediante una descripción visual o a través de las comparaciones con guías de color, a pesar de que el color post-incineración de los dientes no siempre se corresponde con los tonos representados en las mismas (Karkhanis et al.). En nuestro estudio, la descripción visual del color se ha complementado con la utilización de lupas de aumento e imágenes digitales que fueron visualizadas en el programa Adobe Raw ${ }^{\circledR}$, lo que permitió analizar con mayor rigor los cambios de color de las muestras incineradas (Fig. 3). El color de la corona se tornó marrón pálido $\left(100^{\circ} \mathrm{C}\right)$, marrón oscuro $\left(200^{\circ} \mathrm{C}\right)$, marrón grisáceo $\left(400^{\circ} \mathrm{C}\right)$, gris oscuro con manchas azuladas $\left(600^{\circ} \mathrm{C}\right)$, gris oscuro con manchas blancas $\left(800{ }^{\circ} \mathrm{C}\right)$, blanco con manchas grises $\left(1000{ }^{\circ} \mathrm{C}\right) \mathrm{y}$, por último, blanco tiza $\left(1200^{\circ} \mathrm{C}\right)$. Los cambios de coloración en la raíz dental, fueron incluso más homogéneos que a nivel coronal en cada uno de sus grupos, como consecuencia del alto contenido inorgánico que presentó el esmalte (Fig. 3, 4 y 5). Sin embargo, la descripción del color en cada uno de los intervalos de temperatura es diferente según los reportes de unos u otros autores (Muller et al.; Karkhanis et al.) ya que la capacidad para distinguir colores es variable de unas personas a otras, por lo que es necesaria la utilización de metodologías más objetivas y reproducibles como la espectrofotometría o la espectroradiometría. Cuando hemos utilizado la primera para determinar el color (Fig. 9), hemos encontrado que las muestras expuestas a $100^{\circ} \mathrm{C}$ mostraron una disminución marcada en la cromaticidad a*. Dicho descenso tan acusado, no pudo observarse correctamente en un análisis visual, aunque sí logró evidenciarse un tono ligeramente más verdoso (Fig. 3). La luminosidad (L*) disminuyó en los grupos de $200{ }^{\circ} \mathrm{C}$ y $400{ }^{\circ} \mathrm{C}$, lo que se tradujo a nivel visual en una pérdida de brillo, mientras la cromaticidad $\mathrm{b}^{*}$ y el amarilleamiento (YI) se redujeron a los $400{ }^{\circ} \mathrm{C}$ y $600{ }^{\circ} \mathrm{C}$, donde visualmente la corona dental evolucionó al gris azulado y la raíz adquirió un color blanco con manchas grises. En los grupos de temperaturas más altos (800 ${ }^{\circ} \mathrm{C}, 1000{ }^{\circ} \mathrm{C}$ y $1200{ }^{\circ} \mathrm{C}$ ) se fueron incrementando progresivamente los valores de luminosidad $\left(\mathrm{L}^{*}\right)$ y blancura (WI), coincidiendo visualmente con la aparición del color blanco y un aumento del brillo; sin embargo, cromaticidad b* y amarilleamiento (YI) fueron en disminución, observándose estas características reflejadas en el tono azulado que adquirió la corona en un $60 \%$ de las muestras. El cambio de color del diente parece estar relacionado con el rango de temperatura a la que se ha visto sometido y, por tanto, con el grado de incineración y calcinación del diente (Karkhanis et al.; Moreno et al.; Rubio et al., 2015) por lo que el análi- 
sis del color dental resulta de vital importancia en la resolución de casos forenses en los que el fuego se haya visto implicado.

Consideramos que este trabajo de investigación no permite reproducir las condiciones de protección que, en casos reales, le brindan los tejidos blandos labiales y faciales a las coronas dentales; de igual modo, y con respecto a la raíz, este modelo tampoco logró representar las propiedades del hueso alveolar en el que se asienta. Creemos que líneas de investigaciones futuras podrían complementar nuestros resultados, utilizando temperaturas que se instauren de manera más gradual y también más rápida, así como tiempos de exposiciones más cortos; estos modelos permitirán reproducir de manera más cercana la mayoría de circunstancias de estudio en la práctica forense.
El análisis de las alteraciones que sufren los dientes sometidos a altas temperaturas constituye un pilar fundamental para la resolución de casos de interés médico-legal $\mathrm{y}$, particularmente, de aquellos que son de competencia odontológica forense. La marcada resistencia de los dientes a estas vulneraciones hace que, a menudo, éstos sean las pocas evidencias disponibles con información muy valiosa aún en los casos de más complicado abordaje. El estudio de sus alteraciones estructurales y, concretamente, del color dental, es de gran importancia en el examen de cadáveres carbonizados dada la escasez de información aprovechable y con valor médico-legal, por lo que el desarrollo de técnicas viables, objetivas y cuantificables supone un gran avance en el campo forense.

RUBIO, L.; SIOLI, J. M.; SANTOS, I.; FONSECA, G. M. \& MARTIN-DE-LAS-HERAS, S. Morphological changes in teeth exposed to high temperatures with forensic purposes. Int. J. Morphol., 34(2):719-728, 2016.

SUMMARY: Teeth are the most resistant elements of the human skeleton and are thus often used in routine forensic research laboratories. The objective of this study was to describe structural, morphological and color changes that occur post heating the teeth to aid in proper handling of samples in a forensic scenario based on their condition. Seventy teeth were exposed to different temperature intervals, ranging from $100^{\circ} \mathrm{C}$ to $1200^{\circ} \mathrm{C}$ for 60 min using a laboratory oven. Ten unheated teeth were used as controls for the study. After incineration, the teeth were photographed and radiographed to visually assess morphological changes. The measure of color change was performed with a spectrophotometer. The results showed structural and color changes as well as radiographic abnormalities, which were associated to the temperature interval at which the teeth were cremated. In conclusion, it is possible to inform about incineration tooth temperature based on photographic, radiographic and color changes.

KEY WORDS: Burnt tooth; Color changes; X-ray; Morphology; Spectrophotometry.

\section{REFERENCIAS BIBLIOGRÁFICAS}

American Society for Testing and Materials (ASTM). ASTM Document E 313 -73 (Reapproved 1993). Standard Test Method for Indexes of Whiteness and Yellowness of Near-White Opaque Materials. Philadelphia, American Society for Testing and Materials, 1993.

Beach, J. J.; Passalacqua, N. V. \& Chapman, E. N. Heat-Related Changes in Tooth Color: Temperature Versus Duration of Exposure. In: Schmidt, C. W. \& Symes, S. A. (Ed.). The Analysis of Burned Human Remains. London, Academic Press, 2008.

Bonavilla, J. D.; Bush, M. A.; Bush, P. J. \& Pantera, E. A. Identification of incinerated root canal filling materials after exposure to high heat incineration. J. Forensic Sci., 53(2):412$8,2008$.

Fereira, J. L.; Fereira, A. E. \& Ortega A. I. Methods for the analysis of hard dental tissues exposed to high temperatures. Forensic Sci. Int., 178(2-3):119-24, 2008.
Karkhanis, S.; Ball, J. \& Franklin, D. Macroscopic and microscopic changes in incinerated deciduous teeth. J. Forensic Odontoestomatol., 27(2):9-19, 2009.

Martin-de las Heras, S.; Valenzuela, A.; Bellini, R.; Salas, C.; Rubiño, M. \& Garcia, J. A. Objective measurement of dental color for age estimation by spectroradiometry. Forensic Sci. Int, , 132(1):57-62, 2003.

Merlati, G.; Danesino, P.; Savio, C.; Fassina, G.; Osculati, A. \& Menghini, P. Observations on dental prostheses and restorations subjected to high temperatures: experimental studies to aid identification processes. J. Forensic Odontostomatol., 20(2):17-24, 2002.

Merlati, G.; Savio, C.; Danesino, P.; Fassina, G. \& Menghini, P. Further study of restored and un-restored teeth subjected to high temperatures. J. Forensic Odontostomatol., 22(2):34-9, 2004. 
Moreno, S.; Merlati, G.; Marin, L.; Savio, C. \& Moreno, F. Effects of high temperatures on different dental restorative systems: Experimental study to aid identification processes. J. Forensic Dent. Sci., 1(1):17-23, 2009.

Muller, M.; Berytrand, M. F.; Quatrehomme, G.; Bolla, M. \& Rocca, J. P. Macroscopic and microscopic aspects of incinerated teeth. J. Forensic Odontostomatol., 16(1):1-7, 1998.

Myers, S. L.; Williams, J. M. \& Hodges, J. S. Effects of extreme heat on teeth with implications for histologic processing. $J$. Forensic Sci., 44(4):805-9, 1999.

Patidar, K. A.; Parwani, R. \& Wanjari, S. Effects of high temperature on different restorations in forensic identification: Dental samples and mandible. J. Forensic Dent. Sci., 2(1):37-43, 2010.

Paul, S.; Peter, A.; Pietrobon, N. \& Hämmerle, C. H. Visual and spectrophotometric shade analysis of human teeth. J. Dent. Res., 81(8):578-82, 2002.

Piga, G.; Malgosa, A.; Thompson, T. J. U. \& Enzo, S. A New calibration of the XRD technique for the study of archaeological burned human remains. J. Archaeol. Sci., 35(8):2171-8, 2008.

Pol, C. A.; Ghige, S. K.; Gosavi, S. R. \& Hazarey, V. K. Effects of elevated temperatures on different restorative materials: An aid to forensic identification processes. J. Forensic Dent. Sci., 7(2):148-52, 2015

Rubio, L.; Martinez, L. J.; Martinez, E. \& Martin de las Heras, S. Study of short- and long-term storage of teeth and its influence on DNA. J. Forensic Sci., 54(6):1411-3, 2009.

Rubio, L.; Sioli, J. M.; Suarez, J.; Gaitan, M. J. \& Martin-de-lasHeras, S. Spectrophotometric analysis of color changes in teeth incinerated at increasing temperatures. Forensic Sci. Int., 252:193.e1-6, 2015.

Sandholzer, M. A.; Sui, T.; Korsunsky, A. M.; Walmsley, A. D.; Lumley, P. J.\& Landini, G. X-ray scattering evaluation of ultrastructural changes in human dental tissues with thermal treatment. J. Forensic Sci., 59(3):769-74, 2014.

Sandholzer, M. A.; Walmsley, A. D.; Lumley, P. J. \& Landini, G. Radiologic evaluation of heat-induced shrinkage and shape preservation of human teeth using micro-CT. J. Forensic Radiol. Imag., 1(3):107-11, 2013.

Savio, C.; Merlati, G.; Danesino, P.; Fassina, G. \& Menghini, P. Radiographic evaluation of teeth subjected to high temperatures: experimental study to aid identification processes. Forensic Sci. Int., 158(2-3):108-16, 2006.

Shipman, P.; Foster, G. \& Schoeninger, M. Burnt bones and teeth: an experimental study of color, morphology, crystal structure and shrinkage. J. Archaeol. Sci., 11(4):307-25, 1984.
Terada, A. S. S. D.; Araujo, L. G.; Paranhos, L. R.; Silveira, T. C. P.; Pantozzi, G. M. A.; Guimarães, M. A. \& Silva, R. H. A. Orthodontic use of documentation in identification of a skeletonized body in legal dental practice. Int. J. Odontostomat., 8(1):41-6, 2014.

Dirección para Correspondencia:

Prof. Stella Martín de Las Heras

Departamento de Medicina Legal

Toxicología y Antropología Física

Facultad de Medicina

Universidad de Granada

Avenida de la Investigación n 11, C.P. 18016

Granada

ESPAÑA

Email: stella@ugr.es

Recibido: 28-10-2015

Aceptado: 02-02-2016 